\title{
Las Redes I ntegradas de Servicios de Salud desde la realidad cubana
}

\author{
Integrated health service networks on the basis of the Cuban \\ realities
}

\section{Pedro López Puig'; I leana del Rosario Morales Suárez"; Sara Lara Menchaca'I'; Nelcy Martínez Trujillo'v; Santiago Lau Lópezv; Silvio F. Soler Cárdenas"}

\author{
'Especialista de II Grado en Medicina General Integral. Máster en Atención Primaria \\ de Salud Escuela Nacional de Salud Pública. La Habana, Cuba. \\ "Profesora Auxiliar. Escuela Nacional de Salud Pública. La Habana, Cuba. \\ II' Máster en Atención Primaria de Salud. Escuela Nacional de Salud Pública. La \\ Habana, Cuba. \\ IV Licenciada en Enfermería. Máster Salud Pública. Escuela Nacional de Salud \\ Pública. La Habana, Cuba.

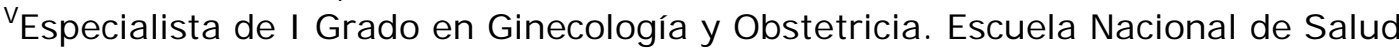 \\ Pública. La Habana, Cuba.
}

\section{RESUMEN}

Introducción En el mes de mayo del año 2008, la Organización Panamericana de la Salud y su Representación en La Habana, a través del Ministerio de Salud Pública cubano, entregó a la Escuela Nacional de Salud Pública la primera versión del Documento de Posición de la Organización sobre las Redes Integradas de Servicios de Salud titulado "Sistemas Integrados de servicios de salud. Conceptos, Opciones de Política y Hoja de Ruta para su implementación en Las Américas. Washington, D.C., 8 de mayo de 2008."

Objetivos Hacer aportes conceptuales, revisión y sistematización de la información y visión crítica del tema.

Métodos La Escuela Nacional de Salud Pública creó, para el cumplimiento del encargo, un grupo de trabajo con profesores propios que en ocho sesiones de trabajo cumplieron el objetivo planteado y aportaron, en las áreas conceptuales, constructos aplicables a la realidad cubana.

Resultados En la red de servicios de Cuba se cumplen los presupuestos de la 
integración propuestos por la OPS. Los conceptos sugeridos en el documento son susceptibles de mejoras estructurales que pueden ser adecuadas a las realidades de cada país.

Conclusiones El tema de las Redes Integradas de Servicios de Salud es de alta complejidad y requiere aproximaciones sucesivas tanto en lo conceptual como en el desarrollo de instrumentos para su medición. Aparentemente, en ningún país o en sus contextos se está totalmente exento de grados variables de integración/fragmentación de sus redes de servicios de salud. La integración de una red de servicios de salud es un proceso no una meta. La determinación del grado y tendencia del par integración/fragmentación en la realidad cubana y el establecimiento de modelos para su estudio son retos para la salud pública del país.

Palabras clave: Redes Integradas de Servicios de Salud, Organización Panamericana de la Salud, Cuba.

\section{ABSTRACT}

Introduction On May 2008, the Pan-American Health Organization and its official headquarters in Havana, through the Ministry of Public Health of Cuba, gave the National School of Public Health the first version of the PAHO Position Document on the Integrated Health Service Systems entitled "Integrated Health Care Systems: Concepts, Political Choices and Waybill for the implementation in the Americas, Washington, D.C, May $8^{\text {th }}, 2008$ ".

Objectives To make conceptual contributions, to review and systematize information and to thoroughly analyze this topic.

Methods For the fulfilment of this task, the National School of Public Health established a working group of professors who, in eight working sessions, were able to attain the set objective and to contribute constructs applicable to the Cuban realities in the conceptual areas.

Results The Cuban health service network puts into practice the PAHO integration assumptions. Regarding the concepts suggested in the document, there is room for structural improvements that may be adapted to the realities of each country.

Conclusions The topic of the Integrated Health Service Networks is highly complex and requires a series of approaches both in the conceptual field and in the development of measuring instruments. Apparently, different levels of integration/fragmentation are present in the health care service networks of every country. The integration of a health service network is a process rather than a target. Determining the level and tendency of the integration/fragmentation pair in the Cuban realities and setting the study models represent challenges for the public health care in the country.

Key words: Integrated health service networks, Pan American Health Organization, Cuba. 


\section{NTRODUCCI ÓN}

El siguiente texto es resultado del análisis sobre la propuesta de la Organización Panamericana de la Salud para alcanzar Redes Integradas de Servicios de Salud en Las Américas. ${ }^{1}$ En su construcción intervinieron un grupo de profesores de la Escuela Nacional de Salud Pública de Cuba que, en ocho sesiones de trabajo, sistematizaron la información, realizaron los señalamientos críticos que consideraron pertinentes y aportaron, en las áreas conceptuales, constructos aplicables a la realidad cubana. Abrir un debate sobre los temas de la Renovación en la Atención Primaria de Salud, ${ }^{2-4}$ y la integración de redes de servicios de salud como parte de las estrategias de renovación desde la perspectiva nacional, parece importante y con amplias posibilidades de desarrollo tanto en el plano conceptual como investigativo.

\section{LA RENOVACIÓN DE LA ATENCIÓN PRI MARIA DE SALUD Y OTROS MANDATOS REGIONALES}

Como bien plantea el documento de la Organización Panamericana de la Salud ${ }^{1}$ existen múltiples instancias de compromisos/mandatos internacionales novinculantes que enfatizan la necesidad de avanzar hacia un cuidado integrado e integral de la salud. ${ }^{5-7}$ Ya en el año 1978, la Declaración de Alma-Atá en su artículo VII sostenía que:

la APS debe mantenerse mediante un sistema integrado, funcional y de sistemas de referencia...., conduciendo al mejoramiento progresivo y comprensivo de la atención sanitaria integral para todos y asignando prioridad a los más necesitados. ${ }^{8}$

En el año 1978 Cuba consolidaba su segunda gran reforma, período o etapa de transformaciones avanzadas en el sector y obtenía resultados sanitarios exitosos a partir de un diseño de servicios enfocados hacia los principales problemas de salud y la garantía de accesibilidad por escalones de atención. ${ }^{9}$

Hacia finales de la década de los 80 la iniciativa de OMS/OPS sobre los Sistemas Locales de Salud (SILOS) también hablaba sobre la necesidad de organizar los servicios de salud en niveles de atención, desarrollar sistemas de referencia y contra-referencia, y hacer una planificación regional de los servicios de salud. ${ }^{10}$ Tan temprano como en el año 1983 Cuba había cumplimentado las metas de Salud para Todos propuestas en Alma-Atá en $1978 .{ }^{11}$ El principal elemento de cambio para que esto sucediera radicaba en los continuos y crecientes esfuerzos que el estado revolucionario realizó a partir de 1959 en el sector de la salud. No obstante los importantes logros obtenidos en el campo sanitario, en el año 1984 se implementa la estrategia sanitaria del Médico y la Enfermera de la Familia, firmemente anclada en la estrategia de atención primaria y como salto cuantitativo y cualitativo en el desarrollo del área de los servicios a la salud y de los recursos humanos. ${ }^{12-15}$

Como bien expresa el documento OPS, el objetivo de organizar servicios de salud en niveles de atención integrados y descentralizados fue nuevamente ratificado por los Estados Miembros en el año 2005 como parte del proceso de renovación de la Atención Primaria de Salud en Las Américas, ${ }^{16-21}$ en el artículo III de la Declaración de Montevideo, que dice que: 
los modelos de atención de salud deben.... fomentar el establecimiento de redes de atención de salud y la coordinación social que vela por la continuidad adecuada de la atención. ${ }^{22}$

Más recientemente, en Junio de 2007, La Agenda de Salud para Las Américas 2008-2017, en su párrafo 49, señala la necesidad de:

fortalecer los sistemas de referencia y contra-referencia y mejorar los sistemas de información a nivel nacional y local de modo de facilitar la entrega de servicios comprensivos y oportunos,...

y en Julio del mismo año, el Consenso de Iquique, logrado en la XVII Cumbre I beroamericana de Ministros de Salud, señala en su párrafo 6,

la necesidad de desarrollar redes de servicios de salud basadas en la atención primaria, de financiamiento público y cobertura universal, dada su capacidad de aminorar los efectos de la segmentación y la fragmentación, articulándose con el conjunto de las redes sociales.

En este contexto Cuba, país pobre y sometido al bloqueo económico y comercial de los EE.UU. por más de 40 años, ha desarrollado un sistema sanitario único, gratuito en todos sus niveles de atención, de cobertura universal, acceso amplio y de sólidas bases en la estrategia de Atención Primaria de Salud, que la coloca en la vanguardia de toda la América en lo que respecta a sus indicadores sanitarios, que constituyen trazadores en la eficacia de un sistema: Mortalidad Infantil, Mortalidad Materna, Esperaza de vida. ${ }^{23-25}$ Todos ellos logrados sin distinción de raza, sexo, nivel económico, filiación política o religiosa.

Se cumplen además los siguientes presupuestos en su red de servicios de salud, tanto transectorial como intersectorialmente:

1. Existe articulación funcional de unidades prestadoras de distinta naturaleza.

2. Se dispone de una organización jerárquica según niveles de complejidad.

3. La división funcional tiene un referente geográfico común y sigue la división político-administrativa de los territorios.

4. Presencia de una dirección única.

5. Normas operacionales, sistemas de información y otros recursos logísticos compartidos.

6. Una misión y visión común a todos sus elementos.

7. El determinante más importante es la voluntad política.

Hasta aquí se puede resumir que:

1. El sistema sanitario cubano tuvo y tiene una estrategia coherente con los fundamentos teóricos de la estrategia de Atención Primaria de salud.

2. Cuba es pionera en el diseño de un sistema sanitario accesible, por niveles coordinados de atención y enfocado hacia los problemas de salud de la población. 


\section{EL DESAFÍ O DE LA FRAGMENTACIÓN DE LOS SERVI CIOS DE SALUD. APLI CACI ONES AL CONTEXTO CUBANO Y APROXI MACIONES CONCEPTUALES}

El primer desafío que presenta la fragmentación de los servicios de salud es considerarla una meta y no un proceso. Realmente se comporta como un par dialéctico integración/fragmentación que presupone un movimiento no lineal. El enfoque estático o como meta y no como proceso, de la integración/fragmentación responde a un pensamiento que no es compatible con un sistema de servicios de cualquier naturaleza y limita su horizonte temporal. El enfoque de par dialéctico permite su visualización en el tiempo en un contexto más complejo y cercano a la realidad y hace que las aproximaciones y evaluaciones sucesivas sean las herramientas indispensables para su estudio cabal. El diseño, implementación y monitoreo continuo de estrategias que permitan visualizar el movimiento del par a través de un gradiente hacia la integración/fragmentación en una red de servicios de salud debe estar firmemente anclado sobre diagnósticos oportunos y precisos.

Se atribuyen además, para su estudio, un conjunto de atributos que hacen complejo el análisis pues cada uno de ellos puede tener un grado y gradiente de integración/fragmentación distinto, por lo que ejercen influencia sobre el sistema y la red de servicios de forma variable.

Como elemento adicional de complejidad cada uno de estos atributos tiene un número variable de elementos constitutivos que pueden llamarse "componentes", que ejercen influencia variable en el movimiento interno de cada atributo hacia la fragmentación o hacia la integración.

Otro elemento a considerar es que no debe separase para su estudio la fragmentación de los servicios de salud de la segmentación de los sistemas de salud al ser fuente de confusión la vinculación semántica de ambos términos así como las complejas interrelaciones de uno con el otro. La segmentación y fragmentación de servicios constituyen barreras de alta significación para el logro de un sistema integrado. No considerarlo así dejaría brechas conceptuales importantes para el diseño de estrategias integradoras.

El desplazamiento del gradiente integración/fragmentación hacia mayor fragmentación de los servicios de salud es, como bien plantea el documento, una causa importante pero aún no suficientemente demostrada del bajo desempeño de los servicios de salud, y por ende del bajo desempeño general de los sistemas de salud.

En la experiencia cubana y partiendo de un diseño e implementación altamente integrado del sistema sanitario, la fragmentación ha estado vinculada de una forma u otra a dificultades en el acceso oportuno a los servicios, la entrega de servicios de cuestionable calidad técnica, el uso irracional e ineficiente de los recursos disponibles, el incremento innecesario de los costos de atención a la salud y la baja satisfacción de los usuarios con los servicios recibidos.

Entre las causas de fragmentación en el medio sanitario cubano se pueden mencionar la falla en los sistemas de referencia y contra-referencia, las dificultades con la conducción estandarizada de la información sobre los pacientes a través del sistema de servicios a la salud, entre otras posibles; por otro lado, la incidencia de la fragmentación en las redes de servicios ha provocado la duplicación de exámenes 
de laboratorio, aumento de los costos con afectación en la eficiencia del sistema, pérdida de tiempo en la gestión de recursos y, por lo tanto, impactos negativos a la economía nacional.

Dentro del campo conceptual hay temáticas que parecen ser medulares por su peso en la formación del esquema mental de los que toman decisiones en las políticas de salud. Entre ellas se consideran las siguientes:

\section{Definición de la OMS de Servicios de Salud I ntegrados}

La gestión y entrega de servicios de salud de forma tal que las personas reciban un continuo de servicios preventivos y curativos, de acuerdo a sus necesidades a lo largo del tiempo y a través de los diferentes niveles del sistema de salud.

De acuerdo al desarrollo alcanzado en Cuba en su estrategia de Atención Primaria de Salud el concepto aportado por la OMS deja brechas de actuación del personal de la salud que son necesarias incluir, así como omite elementos de calidad que deben ser tomados en cuenta.

\section{Propuesta cubana}

La gestión y entrega de servicios de salud de forma tal que las personas reciban un continuo de servicios de promoción, prevención, diagnóstico, curación, rehabilitación y reinserción social; de acuerdo a sus necesidades, a lo largo del tiempo y a través de los diferentes niveles del sistema de salud, con una eficiencia, eficacia y efectividad aceptables para el momento tecnológico e histórico social con que se cuente

\section{Fundamentación}

1. Explicita mejor los campos de integración desde la promoción hasta la reinserción social.

2. Introduce la temática de la calidad desde tres aproximaciones: eficiencia, eficacia y efectividad.

3. Establece un rasero de aceptabilidad que induce a considerar la evaluación continua como parte del proceso.

4. Ubica temporalmente de acuerdo a los condicionamientos socio-históricos y tecnológicos los componentes de calidad.

\section{Crítica a la definición de sistema integrado de servicios de salud, del profesor Shortell. Universidad de Northwestern, EE.UU.}

El profesor Stephen Shortell define un sistema integrado de servicios de salud como:

Una red de organizaciones que presta o hace los arreglos para prestar un continuo coordinado de servicios de salud a una población definida, y que está dispuesta a rendir cuentas por sus resultados clínicos y económicos y por el estado de salud de la población a la que sirve. 


\section{Comentarios}

1. El concepto presupone la posibilidad de lograr integración en sistemas segmentados o fragmentados.

2. La posibilidad de lograr en un marco temporal aceptable, dentro del contexto Latinoamericano, sistemas integrados sin reducir o transformar los altos niveles de fragmentación y/o segmentación, parece improbable.

3. Se establece la coordinación como meta en vez de la integración desde los valores y compromisos compartidos por las partes. Con lo anterior no se resta ningún valor a la importancia de las relaciones contractuales como elementos facilitadores de la coordinación que siempre será necesaria y esta implícita en el proceso de integración.

4. La aceptación de este concepto implica dejar brechas demasiado anchas a la posibilidad de continuar desarrollando políticas segmentadas y/o fragmentadas en la oferta de servicios con un supuesto enfoque integrador.

\section{Definición de OPS de fragmentación de los servicios de salud}

Dice la definición: "coexistencia de muchas unidades o entidades no integradas en la red de servicios de salud". Salud en las Américas 2007, Washington DC: OPS/OMS.

\section{Comentarios}

1. Definición demasiado general. No permite visualizar claramente que se persigue, para qué y por qué.

2. Induce a error de juicio al limitar la fragmentación a la no integración en la red de servicios de las unidades. Es decir, no discrimina niveles intermedios y se pierde de vista el proceso.

3. No queda claro que es la "no integración."

4. Lo negativo en la tendencia se manifiesta porque no está lo positivo, pero esto último no está explícitamente esclarecido.

\section{Propuesta cubana}

Fragmentación de los servicios de salud: "el nivel inferior de aprovechamiento de la estructura y los procesos que tienden a garantizar un continuo asistencial integral a cada problema de salud.

\section{Fundamentación}


1. Introduce el necesario tema de la evaluación del proceso como única vía para determinar avances o retrocesos y rectificar las hojas de ruta de cada país.

2. Reconoce la tendencia natural de las relaciones sociales a crear mecanismos de coordinación de procesos vinculados.

3. Introduce el término de continuo-asistencial-integral como camino posible y necesario para el manejo de las demandas de salud sea cual sea su naturaleza

En conclusión, el tema de las Redes Integradas de Servicios de Salud es de alta complejidad y requerirá aproximaciones sucesivas tanto en lo conceptual como en el desarrollo de instrumentos para su medición. Aparentemente, en ningún contexto de país se está totalmente exento de grados variables de integración/fragmentación de sus redes de servicios de salud. La integración de una red de servicios de salud es un proceso no una meta. La determinación del grado y tendencia del par integración/fragmentación en la realidad cubana y el establecimiento de modelos para su estudio son retos para la salud pública del país.

\section{REFERENCI AS BI BLI OGRÁFI CAS}

1. Organización Panamericana de la Salud/ Organización Mundial de la Salud. Sistemas Integrados de Servicios de Salud. Conceptos, Opciones de Política y Hoja de Ruta para su implementación en las Américas. Washington, D.C.: OPS/OMS;2008. [8 de mayo de 2008].

2. Roses Periago M. La renovación de la atención primaria de salud en las Américas: la propuesta de la Organización Panamericana de la Salud para el siglo xxi. Rev Panam Salud Pública. 2007;21(2/3):65-9.

3. Macinko J, Montenegro H, Nebot Adell C, Etienne C. Grupo de Trabajo de Atención Primaria. La renovación de la atención primaria de salud en las Américas. Rev Panam Salud Pública. 2007;21(2/3):73-84.

4. Renovación de la Atención Primaria de Salud en Las Américas. Washington: OPS,2005. [Mayo 2005: Cap. 1, 6 y Cap. 8].

5. Integración de la Atención a la Salud. Informe de un grupo de Estudio de la OMS. Ginebra: OMS; 1997. [OMS. Serie de Informes Técnicos, No 861].

6. World Health Organization. Integrated health services-what and why? Geneva: WHO; 2008. [Technical Brief No. 1].

7. Lega F. Organisational design for health integrated delivery systems: theory and practice. Health Policy. 2007; 81:258-79.

8. World Health Organization. Atención Primaria de Salud. Informe de la Conferencia Internacional sobre Atención Primaria de Salud. Geneva: WHO; 1978. [Alma-Atá, URSS, 6-12 de septiembre de 1978].

9. Delgado García G. Desarrollo histórico de la salud pública cubana. Rev Cubana Salud Pública. 1998;24(2): 110-8. 
10. Los sistemas locales de salud. Conceptos, métodos, experiencias [sitio en Internet]. [citado May 2009]. Disponible en:

http:// publications.paho.org/ product.php? productid $=95 \& j s=n$

11. Ministerio de Salud Pública de Cuba (MINSAP). Fundamentación para el nuevo enfoque de la Medicina en la Comunidad. La Habana: MINSAP; 1975.

12. El Plan del Médico de la Familia en Cuba. UNICEF. UNFPA. OPS. OMS. La Habana: MINSAP; 1991.

13. Ministerio de Salud Pública de Cuba (MINSAP). Resumen del Proceso de Implementación de la Carpeta Metodológica de Atención Primaria de Salud en los grupos básicos de trabajo (GBT) de Ciudad de La Habana. La Habana: MINSAP, Dirección Nacional de Servicios Ambulatorios; 2001.

14. . Los programas de Epidemiología en la Medicina General Integral. La Habāna: MI NSAP; 1985.

15. MI NS̄ĀP; 1986.

Programa de Atención Integral al Adulto. MINSAP. La Habana:

16. Giovanella L. A Atenção Primária à saúde nos países da União européia: configurações e reformas organizacionais na década de 1990. Cad. Saúde Pública. 2006; 22(5): 951-63.

17. Homedes N, Ugalde A. Human resources: the Cinderella of health sector reform in America. Hum Resources Health [serie en Internet]. 2005 Jan [citado 23 Mar 2007]; 3(1). Disponible en: http://www. human-resourceshealth.com/content/3/1/1

18. Murray CJ L, Frenk J. A framework for assessing the performance oh health systems. Bull WHO. 2000;78(6):717-31.

19. Fritzen SA. Strategic management of the health workforce in developing countries: what have we learned? Hum Resources Health [serie en Internet]. 2007 Feb [citado 23 Mar 2007]; 5(4). Disponible en: http://wwww.human-resourceshealth.com/content/5/1/4

20. Glendinning C, Rummery K, Clarke R. From collaboration to commissioning: developing relationship between primary health and social services. BMJ . 1998; 317(11): 122-5.

21. Lethbridge $J$. Public sector reform and demand for human resources for health Humn Resources Health [serie en Internet]. 2004 Nov [citada 23 Mar 2007]; 2(5). Disponible en : http://wwww.human-resources-health.com/content/2/1/15

22. Consulta regional sobre la renovación de la atención primaria de salud (APS) en Las Américas. Montevideo, Uruguay: OPS/OMS;2005 [sitio en Internet]. [citado 2009 May]. Disponible en:

http://www.paho.org/Spanish/AD/THS/OS/aps_agenda.htm

23. Ministerio de Salud Pública de Cuba (MINSAP). Resumen de los problemas detectados durante las inspecciones territoriales del MI NSAP al área de la Atención 
Primaria de Salud. Años 1998,1999, 2000. La Habana: MINSAP, Dirección Nacional de Servicios Ambulatorios; 2001.

24. . Los Programas de Salud: Concepción y Evaluación a Nivel de la Atención Primaria de Salud. La Habana: MINSAP, Área de Higiene y Epidemiología; 2000.

25. El Programa de Atención Médica Integral a la Familia. La Habana: MI NSAP, Dirección Nacional de Atención Ambulatoria; 1987.

Recibido: 6 de enero de 2009.

Aprobado: 28 de marzo de 2009.

Pedro López Puig. Escuela Nacional de Salud Pública. Calle Línea esq. a I. El Vedado 10400. La Habana, Cuba. E-mail: plpuig@infomed.sld.cu 